\title{
Extended Interplanar Linking in Graphite Formed from Vacancy Aggregates
}

\author{
T. Trevethan, P. Dyulgerova, C. D. Latham, and M. I. Heggie \\ Department of Chemistry, University of Surrey, Guildford, Surrey GU2 7XH, United Kingdom \\ C. R. Seabourne and A. J. Scott \\ Institute for Materials Research, SPEME, University of Leeds, Leeds LS2 9JT, United Kingdom \\ P. R. Briddon \\ School of Electrical and Electronic Engineering, University of Newcastle upon Tyne, Newcastle NE1 7RU, United Kingdom \\ M. J. Rayson \\ Institutionen för matematik, Luleå Tekniska Universitet, SE-97187 Luleå, Sweden
}

(Received 19 March 2013; published 27 August 2013)

\begin{abstract}
The mechanical and electrical properties of graphite and related materials such as multilayer graphene depend strongly on the presence of defects in the lattice structure, particularly those which create links between adjacent planes. We present findings which suggest the existence of a new type of defect in the graphite or graphene structure which connects adjacent planes through continuous hexagonal $s p^{2}$ bonding alone and can form through the aggregation of individual vacancy defects. The energetics and kinetics of the formation of this type of defect are investigated with atomistic density functional theory calculations. The resultant structures are then employed to simulate high resolution transmission electron microscopy images, which are compared to recent experimental images of electron irradiation damaged graphite.
\end{abstract}

DOI: 10.1103/PhysRevLett.111.095501

PACS numbers: 61.72.Bb, 61.72.Lk, 61.80.Hg, 73.22.Pr

The rich variety of structures and topologies that result from the ways that carbon atoms can bond with each other provides us with a multitude of opportunities and challenges. A particularly important problem concerns the response of graphite to damaging radiation, especially where it forms the neutron moderator and reflector blanket in a nuclear fission reactor. In this application, it is essential for the structural integrity of the material to be maintained for decades in a harsh, inaccessible environment. The mechanical and electrical properties of graphite, as well as other forms of nanostructured carbon and graphene, can be substantially altered through irradiation [1-3]. In the case of graphite, irradiation (with neutrons, electrons, or ions) causes changes to electrical resistance and thermal conductance $[2,4,5]$ and anisotropic changes to both elastic properties and the crystal dimensions, together with expansion in the prismatic direction, balanced by shrinkage in the basal plane [6,7]. These changes arise from a complex evolution of a population of point defects in the graphite structure (Frenkel pairs of lattice vacancies and self-interstitials created when impacting energetic particles displace atoms) into prismatic and basal dislocations [8] and other interstitial and vacancy aggregates [9-11]. The processes involved occur over many time and length scales, and the fundamental mechanisms behind the observed property changes are still unresolved, even after 70 years of intensive research.

One of the most useful experimental insights into the evolution of the graphite structure under irradiation comes from high resolution transmission electron microscopy (HRTEM) images [12]. This method can resolve prismatic edge and basal dislocations in the graphite structure when viewing along the $c$ axis (i.e., perpendicular to the planes) and the topology of individual layers and prismatic edge dislocations when viewing along the planes (side on) [13]. HRTEM images typically show evidence for the rupturing and bending of the planes $[14,15]$ as well as dislocation climb and the growth of new planes under irradiation [16]. However, the atomistic mechanisms driving these processes are not revealed, since such images are unlikely to be able to see individual atomic positions and point defects in the basal direction, and it has thus far proven impossible to observe a single interstitial between planes [17].

To gain a fundamental insight into the atomistic processes that drive the overall behavior of the material, chemically accurate $a b$ initio simulations, in this case at the level of density functional theory (DFT), can be employed. DFT calculations show that the ground state for the single interstitial defect is the spiro structure [18], which forms strong bonds of $s p^{3}$ character to the adjacent carbon sheets, and thus inhibits relative translation of the layers, resulting in an increase to the shear modulus, and potentially increases the $c$-axis electrical conductivity $[11,19]$.

In its ground state, the single vacancy undergoes a spontaneous Jahn-Teller distortion [20], resulting in a bond forming between two of its three neighbouring carbon atoms which can reorientate via a small barrier [21]. The present consensus is that single vacancies can migrate 
(a)

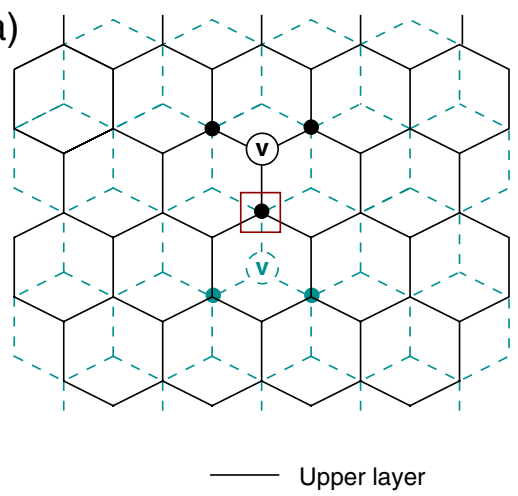

(b)

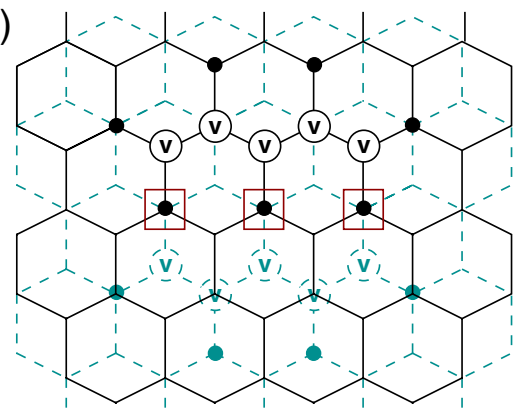

(v) Vacancy (c)

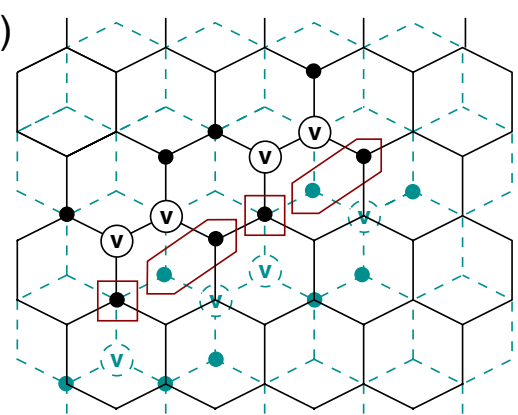

- 2 coordinated atom

FIG. 1 (color online). (a) Schematic of the $V_{2}^{2} \beta \beta$ interlayer divacancy, where the red square highlights the two under coordinated atoms that form the interlayer bond. (b) Two $V_{5}$ lines forming three interlayer bonds. (c) Extending $V_{2}^{2} \beta \beta$ in the armchair direction, with two $V_{4}$ lines forming four interlayer bonds.

with an activation energy of approximately $1.1 \mathrm{eV}$, meaning they would be mobile at temperatures above $100{ }^{\circ} \mathrm{C}-200^{\circ} \mathrm{C}$ [22]. Two vacancies in neighboring planes can bind to form stable interlayer divacancy aggregates, pinning adjacent layers in a similar way to the spiro interstitial [11,22]. In the case of vacancies in $\beta$ sites neighboring each other in adjacent layers (the $V_{2}^{2} \beta \beta$ divacancy in the notation of Ref. [11]) [Fig. 1(a)] where two unbonded atoms are situated directly above one another, the formation of the interlayer bond releases $3.4 \mathrm{eV}$ $[11,22]$. This is much more energy than the single vacancy migration barrier and the spiro interstitial migration barrier of $\approx 2 \mathrm{eV}$ [23]. The deep potential well created by the $V_{2}^{2} \beta \beta$ interlayer divacancy means that once formed, it will become immobile (at temperatures below $\approx 1000{ }^{\circ} \mathrm{C}$ ) and act as a nucleation site for further aggregation of vacancies. We propose that this aggregation can lead to further interlayer bonding from dangling bonds at the edges of larger vacancy aggregates, resulting in an extended interlayer defect, altering the topology of the graphite structure. In this Letter, we describe calculations which demonstrate how this dislocation is formed and present simulated HRTEM images which indicate that this structural motif has been observed experimentally in irradiated graphite.

The formation of the extended defects from an aggregation of mobile single vacancies in the graphite structure occurs as follows. From the $V_{2}^{2} \beta \beta$ structure in Fig. 1(a), by removing a further two atoms in each layer in the zigzag direction to create two three-atom vacancies, an additional $s p^{2}$ interlayer bond can be formed. By then removing two further atoms from each layer in the same direction, another interlayer bond can be formed [Fig. 1(b)], and so on. As the vacancy lines are extended, so is the interlayer binding, resulting in the topology illustrated in Fig. 2(a). Here, it can be seen that the interlayer binding leads to the upper plane joining the lower plane in a continuous, graphene ribbon. This structure can also be viewed as a dipole of two screw dislocations (the edges of the ribbon) in opposite directions connected with two prismatic edge dislocations [Fig. 2(b)], i.e., a loop of partial dislocation with Burgers vector $\mathbf{b}=\mathbf{c}$.

As well as creating two vacancy lines in the zigzag direction, the vacancies of the $V_{2}^{2} \beta \beta$ can be extended in the armchair direction, again forming an extended interlayer connection forming further bonds [Fig. 1(c)]. However, in this case, the commensuration between the vacancy lines in adjacent layers ( $A B$ graphite) is different, and more local distortion of the structure and/or basal shear is required for the interlayer bonds to form. Moreover, only one additional vacancy is needed to be added to the $V_{2}^{2} \beta \beta$ structure in each layer to form an additional interlayer bond.

To investigate the formation of these defects, DFT calculations were performed using the AIMPRO program package [24] at the local density approximation [25] (with activation energies confirmed with the generalized gradient approximation [26]) employing Hartwigsen-GoedeckerHutter pseudopotentials [27]. The graphite crystal is represented with orthorhombic supercells containing four layers each containing 72 atoms. The method is explained in detail elsewhere [19]. The total energies of the complexes do not change significantly for larger system sizes; however, the in-plane reconstruction of the longer isolated vacancy lines is sensitive to the cell size: calculations with 128 atoms per layer show that this can lower the energy of the in-plane reconstructions by 0.3 and $0.5 \mathrm{eV}$ for the $V_{5}$ and $V_{7}$ lines, respectively. This has no effect on the total formation energies of the interlayer defects (a)

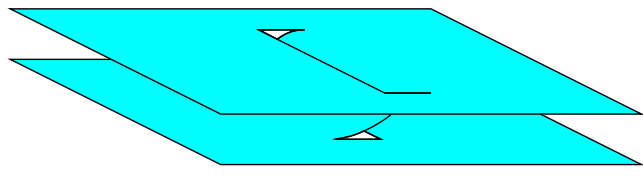

(b)

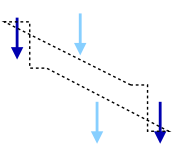

FIG. 2 (color online). (a) Topology of the extended defect and (b) dislocation loop representation (arrows indicate the Burgers vectors of the loop; the dark arrows are screw dislocation vectors, and the light are edge dislocation vectors). 
(a)

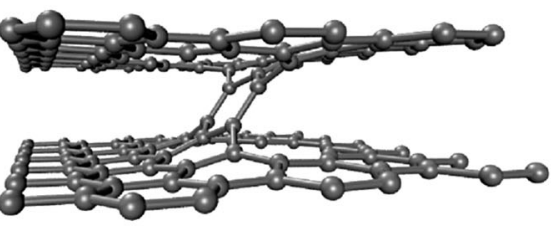

(b)

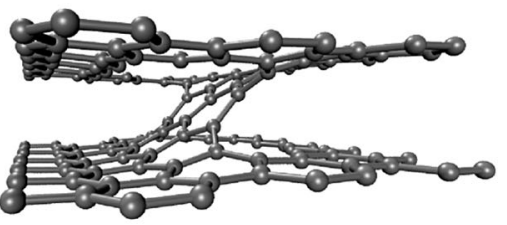

(c)

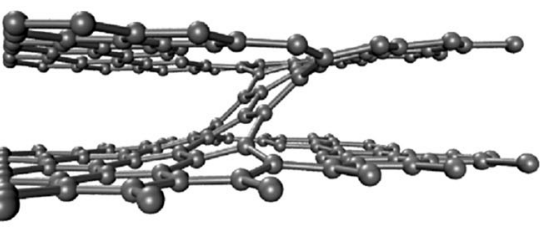

FIG. 3. (a) Optimized structure of the bonded $2 \times V_{3}$ zigzag defect. (b) Optimized structure of the bonded $2 \times V_{5}$ zigzag defect. (c) Optimized structure of the bonded $2 \times V_{3}$ armchair defect.

but will slightly reduce the interlayer binding energies. The coordinates of all atoms are optimized to minimize their total energy, except where constraints are used to prevent basal shear at the edge of the cell.

First, considering the zigzag direction, with two nearest neighbor three-atom vacancy $\left(V_{3}\right)$ aggregates in adjacent layers. The coalescence energy $\left(E_{c}\right)$ (the energy released from the coalescence of three isolated monovacancies in a single layer) for each $V_{3}$ is $12.5 \mathrm{eV}$ (giving $25 \mathrm{eV}$ in total). When two interlayer bonds are formed connecting them, a further $2 \mathrm{eV}$ is released [see Fig. 3(a)]: this is the interlayer bonding energy $\left(E_{b}\right)$, giving a total of $27 \mathrm{eV}$ released from the coalescence of six isolated monovacancies. This bonding generates a relative lateral shift of $\approx 0.3 \AA$ between the two layers. If basal shear is prevented via constraints, then the energy released by the interlayer bonding is reduced by $\approx 0.4 \mathrm{eV}$ (the shearing energy $E_{s}$ ). This interlayer binding energy is significantly less than that for the single bond of the $V_{2}^{2} \beta \beta$ divacancy $(3.4 \mathrm{eV})$, due to the fact that the $V_{3}$ possesses two reconstructed bonds, with one unpaired atom, and these bonds must be broken in order to form the two interlayer bonds (whereas the formation of the $V_{2}^{2} \beta \beta$ does not require breaking of either monovacancy reconstruction [11]).

As the length of the two vacancy lines increases, the energy released per interlayer bond increases due to the energy cost of breaking the pentagons at the end of the lines taking a smaller proportion of the balance, and the energy per bond approaches that of the single bond of the $V_{2}^{2} \beta \beta$ (see Table I). The basal shear induced by the interlayer bonding converges to $0.6 \AA$ as the length of the defect increases, which maximizes the potential energy from the

TABLE I. Energetics of different length zigzag (ZZ) and armchair (AC) direction interlayer defects. $n_{b}$ is the number of interlayer bonds, $E_{c}$ the coalescence energy $(\mathrm{eV}), E_{b}$ the interlayer bonding energy $(\mathrm{eV}), E_{s}$ the shear energy $(\mathrm{eV})$, and $\Delta S$ the shear displacement $(\AA)$.

\begin{tabular}{lcrrccc}
\hline \hline Defect & $n_{b}$ & \multicolumn{1}{c}{$E_{c}$} & \multicolumn{1}{c}{$E_{b}$} & $E_{b} / n_{b}$ & $E_{s}$ & $\Delta S$ \\
\hline$V_{2}^{2} \beta \beta$ & 1 & 0.1 & 3.4 & 3.4 & 0.0 & 0.0 \\
ZZ $V_{3}-V_{3}$ & 2 & 25.0 & 2.0 & 1.0 & 0.4 & 0.3 \\
ZZ $V_{5}-V_{5}$ & 3 & 42.2 & 6.5 & 2.2 & 0.5 & 0.6 \\
ZZ $V_{7}-V_{7}$ & 4 & 61.7 & 11.8 & 3.0 & 1.3 & 0.6 \\
AC $V_{2}-V_{2}$ & 2 & 9.9 & 2.2 & 1.1 & 0.4 & 0.5 \\
AC $V_{3}-V_{3}$ & 3 & 18.7 & 3.1 & 1.0 & 1.3 & 0.9 \\
\hline \hline
\end{tabular}

formation of the interlayer bonds. This displacement will introduce a localized stacking fault, moderated by the elastic response of the surrounding material.

In the case of the armchair direction, for a pair of divacancies, the energy released upon the formation of the two interlayer bonds is $2.2 \mathrm{eV}$ (1.1 eV per bond) and resulting in a relative basal shear of $\approx 0.5 \AA$. Constraining this shear reduces the bonding energy by $0.4 \mathrm{eV}$ (to $1.8 \mathrm{eV}$ ). The effect of extending both lines is shown in Table I. The energy released per interlayer bond is less than for the zigzag direction: this is due to the fact that the unbound lines "heal" to saturate dangling bonds, and there is an energy cost in breaking this. The healing, or collapse, of the zigzag direction lines causes a much larger strain in the surrounding layer and does not become energetically favorable until the lines are much longer. For the zigzag direction, $2 n-1$ vacancies are required to form $n$ interlayer bonds, whereas in the armchair direction, only $n$ vacancies are required [see Fig. 1(c)]. Even so, with the formation energy of a single vacancy of $7.9 \mathrm{eV}$ [22], it is clear from Table I that the zigzag direction defect is energetically favored, at least for relatively short lines.

The formation of the interlayer defect in both directions is energetically favored over the corresponding isolated vacancy lines, long believed to form from vacancy aggregation in the standard model of radiation damage of graphite [28,29], and which have been observed in HRTEM images of graphene [30]. However, their formation from a given population of mobile vacancies in the graphite structure and the overall favored direction will depend on kinetic factors. The interlayer defects will be nucleated by the initial formation of the $V_{2}^{2} \beta \beta$ divacancy, which will have an activation barrier to form when two diffusing single vacancies in adjacent layers become coincident. We calculated this barrier, using the nudged elastic band method [31,32], to be only $0.3 \mathrm{eV}$, which would be activated almost instantaneously at the temperatures where monovacancies are mobile. Once formed, additional mobile vacancies can coalesce to this defect and extend it. Once an extended defect has been established, additional mobile vacancies can either (i) diffuse to the ends of the vacancy lines in each layer, extending the defect and the interlayer bonding; (ii) diffuse to the unbonded side of the vacancy lines, resulting in climb or glide of the prismatic dislocation edges (Fig. 2); or (iii) diffuse into the graphene ribbon connecting the layers, resulting 
in the defect contracting or shortening. A complete determination of the potential energy landscape of the aggregation process is required to obtain a full quantitative understanding of the kinetic behavior of the system, but initial calculations show that the barriers for the final step of coalescence of a vacancy to the end of the interlayer defect lines are lowered by up to $0.6 \mathrm{eV}$ (relative to inplane vacancy coalescence [22]) due to the strain caused by the interlayer binding. This coalescence mechanism will be in competition with purely in-plane coalescence processes, forming the 5-8-5 divacancy and higher order complexes. However, even after in-plane multivacancies have been formed, it is still possible for mobile monovacancies in neighboring planes to react with these.

Further calculations have demonstrated that, once formed, these dislocations can be kinetically stable up to relatively high temperatures, especially if there is any climb of the vacancy line edges. There are two possible structural changes away from the stable potential energy minimum occupied by the dislocation. The first is to break the interlayer bonds and return to a pair of vacancy lines; however, the activation barriers for this will be very high. It is also possible that atoms forming part of the interlayer bond move between the layers, leaving one undefective plane and a larger vacancy loop in the other layer (which will release a substantial amount of energy). For example, the structure in Fig. 3(a) can transform to a $V_{6}$ loop in a single plane. The barrier to this process can be less than $1 \mathrm{eV}$ (and occur at room temperature) if both vacancy lines in adjacent layers are of identical length. If they are not, or if there is climb of the unbonded edge of either line (from further vacancy coalescence), then the barriers for this change are much higher $(>2.5 \mathrm{eV})$. It is not yet clear what will be the effect of interstitial atoms interacting with this structure, which become mobile at higher temperatures $\left(\approx 500{ }^{\circ} \mathrm{C}\right)$. If they encounter the defect, then they may either heal it or grow the unbound prismatic edges outward (glide) and nucleate new basal planes.

To investigate the typical contrast these type of structures would generate in HRTEM, we employed the HRTEM module of the CERIUS2 package to simulate imaging, employing a multislice methodology. The accelerating voltage parameter was set to $200 \mathrm{kV}$ (as in Ref. [16]), and a spherical aberration coefficient of $1.2 \mathrm{~mm}$ was used. Thickness-defocus plots were found for the simulated structures, and the simulated images are for ultrathin theoretical specimens $3 \mathrm{~nm}$ in thickness. Figure 4(a) shows a simulated image for the structure of a $V_{7}-V_{7}$ interlayer defect in the zigzag direction at a defocus of $\approx 700 \AA$. This closely resembles Fig. 4(b) taken from a section of a noise filtered HRTEM image of electron irradiated graphite, in a recent study by Karthik et al. [16], which was observed frequently appearing, disappearing, and undergoing dislocation climb. One notable feature in the experimental image is the curvature of the planes above and below the defect, which is (a)

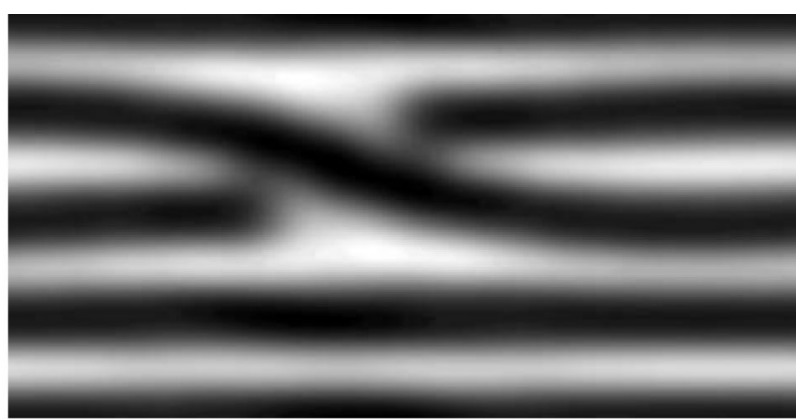

(b)

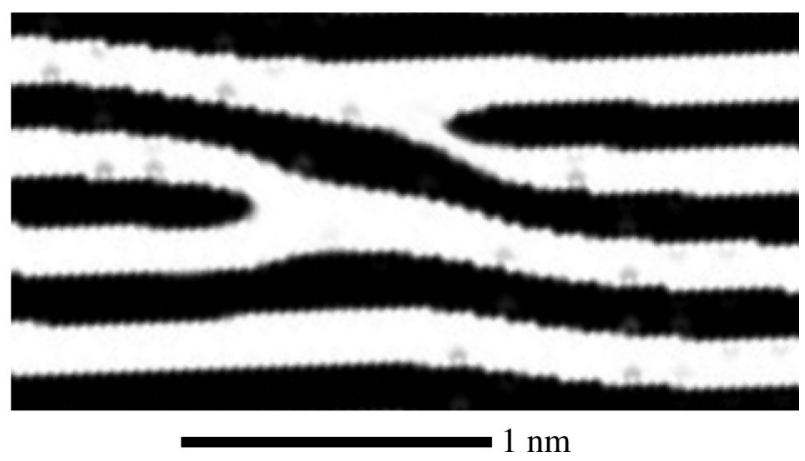

FIG. 4. (a) Simulated HRTEM image of a $V_{7}-V_{7}$ interlayer defect viewing parallel to the line of the defect. (b) Experimental HRTEM image of irradiated graphite, reprinted with permission from C. Karthik et al., J. Nucl. Mater. 412, 321 (2011). Copyright 2011, Elsevier.

not reproduced in our calculations: this is most likely due to the elastic response of the surrounding crystal to the changing interplanar separation across the defect. Other experimental studies have found similar structures occurring in pyrolytic carbon undergoing graphitization [33]. In summary, we have demonstrated that stable extended defects can occur in graphite and multilayer graphene that link adjacent planes with only $s p^{2}$ bonds and that they can form from an aggregation, or assembly, of vacancy point defects. This type of defect will have significant effects on the behavior of the material and will prevent basal shear and pin basal dislocations, changing the elastic properties.

The authors wish to thank EdF Energy and the EPSRC for providing financial support. The views expressed in this work are independent from those which may be held by our sponsors.

[1] J.H.W. Simmons, Radiation Damage in Graphite (Pergamon, New York, 1965).

[2] B. T. Kelly, Physics of Graphite (Applied Science, Barking, NJ, 1981).

[3] F. Banhart, Rep. Prog. Phys. 62, 1181 (1999).

[4] M. Burton and T. J. Neubert, J. Appl. Phys. 27, 557 (1956).

[5] T. Iwata, T. Nihira, and H. Matsuo, J. Phys. Soc. Jpn. 36, 123 (1974).

[6] Chemistry and Physics of Carbon, edited by P. L. Walker (Edward Arnold, London, 1966), Vol. 2. 
[7] B. T. Kelly, B. J. Marsden, K. Hall, D. G. Martin, A. Harper, and A. Blanchard, Irradiation Damage in Graphite due to Fast Neutrons in Fission and Fusion Systems, TECDOC Vol. 1154 (IAEA, Vienna, 2000).

[8] M. I. Heggie, I. Suarez-Martinez, C. Davidson, and G. Haffenden, J. Nucl. Mater. 413, 150 (2011).

[9] R. H. Telling and M. I. Heggie, Philos. Mag. 87, 4797 (2007).

[10] E. Kaxiras and K. C. Pandey, Phys. Rev. Lett. 61, 2693 (1988).

[11] R. H. Telling, C. P. Ewels, A. A. El-Barbary, and M. I. Heggie, Nat. Mater. 2, 333 (2003).

[12] D. B. Williams and C. B. Carter, Transmission Electron Microscopy: A Textbook for Materials Science (Springer, New York, 2009), 2nd ed.

[13] P. A. Thrower, Carbon 6, 687 (1968).

[14] T. Tanabe, Phys. Scr. T64, 7 (1996).

[15] A. Asthana, Y. Matsui, M. Yasuda, K. Kimoto, T. Iwata, and K. Ohshima, J. Appl. Crystallogr. 38, 361 (2005).

[16] C. Karthik, J. Kane, D. P. Butt, W. E. Windes, and R. Ubic, J. Nucl. Mater. 412, 321 (2011).

[17] C. R. Seabourne, R. Brydson, M. I. Heggie, C. D. Latham, and A. J. Scott, J. Phys. Conf. Ser. 371, 012061 (2012).

[18] M. I. Heggie, B. R. Eggen, C. P. Ewels, P. Leary, S. Ali, G. Jungnickel, R. Jones, and P. R. Briddon, in Fullerenes: Chemistry, Physics, and New Directions, Recent Advances in the Chemistry and Physics of Fullerenes and Related Materials Vol. 6, edited by K. M. Kadish and R. S. Ruoff (The Electrochemical Society, Pennington, NJ, 1998) p. 60.
[19] C. D. Latham, M. I. Heggie, J. A. Gámez, I. SuárezMartínez, C.P. Ewels, and P.R. Briddon, J. Phys. Condens. Matter 20, 395220 (2008).

[20] C. A. Coulson, M. A. Herraez, M. Leal, E. Santos, and S. Senent, Proc. R. Soc. A 274, 461 (1963).

[21] A. A. El-Barbary, R. H. Telling, C. P. Ewels, M. I. Heggie, and P. R. Briddon, Phys. Rev. B 68, 144107 (2003).

[22] C. D. Latham, M. I. Heggie, M. Alatalo, S. Öberg, and P. R. Briddon, J. Phys. Condens. Matter 25, 135403 (2013).

[23] H. Zhang, M. Zhao, X. Yang, H. Xia, X. Liu, and Y. Xia, Diam. Relat. Mater. 19, 1240 (2010).

[24] P. R. Briddon and R. Jones, Phys. Status Solidi B 217, 131 (2000).

[25] J. P. Perdew and Y. Wang, Phys. Rev. B 45, 13244 (1992).

[26] J. P. Perdew, K. Burke, and M. Ernzerhof, Phys. Rev. Lett. 77, 3865 (1996).

[27] C. Hartwigsen, S. Goedecker, and J. Hutter, Phys. Rev. B 58, 3641 (1998).

[28] B. T. Kelly, Carbon 20, 3 (1982).

[29] N. Gallego and T.D. Burchell, Oak Ridge National Laboratory Technical Report No. ORNL/ TM-2011/378, 2011 [http://pbadupws.nrc.gov/docs/ ML1131/ML113110373.pdf].

[30] A. W. Robertson, C. S. Allen, Y. A. Wu, K. He, J. Olivier, J. Neethling, A.I. Kirkland, and J.H. Warner, Nat. Commun. 3, 1144 (2012).

[31] G. Henkelman, B. P. Uberuaga, and H. Jónsson, J. Chem. Phys. 113, 9901 (2000).

[32] G. Henkelman and H. Jónsson, J. Chem. Phys. 113, 9978 (2000).

[33] J.-M. Leyssale, J.-P. Da Costa, C. Germain, P. Weisbecker, and G. L. Vignoles, Carbon 50, 4388 (2012). 